\title{
A DECISION BASED CRITERION FOR SELECTING PARAMETERS IN \\ EVALUATION OF PEDESTRIAN SAFETY PROBLEMS WITH THE TRAFFIC CONFLICT ANALYSIS TECHNIQUE.
}

\author{
by \\ Hugo Pietrantonio, D.Sc. * \\ Transportation Engineer, Professor \\ Department of Transportation Engineering \\ Polytechnic School - University of São Paulo, Brazil \\ Edifício de Engenharia Civil, Cidade Universitária \\ São Paulo/SP, CEP 05508-900, Brazil \\ Email: hpietran@usp.br or hugo.pietrantonio@poli.usp.br \\ Phone: +55-11-3091-5512; Fax: +55-11-3091-5716 \\ * Corresponding Author \\ and \\ Luiz Fernando Bizerril Tourinho, EIT, M.Sc. \\ Master of Science Student \\ Department of Transportation Engineering \\ Polytechnic School - University of São Paulo, Brazil \\ Edifício de Engenharia Civil, Cidade Universitária \\ São Paulo/SP, CEP 05508-900, Brazil \\ Email: sp4u@usa.net or sp4u@ bol.com.br \\ Phone: +55-11-3091-5492; Fax: +55-11-3091-5716
}

\author{
Submitted for publication in the journal \\ Transportation Planning and Technology
}

November 2004

Key words: traffic conflicts, pedestrian safety, urban signalized intersections.

Word count: 5928 words +10 figures/tables. 


\title{
A DECISION BASED CRITERION FOR SELECTING PARAMETERS IN \\ EVALUATION OF PEDESTRIAN SAFETY PROBLEMS WITH THE TRAFFIC CONFLICT ANALYSIS TECHNIQUE.
}

Hugo Pietrantonio and Luiz F.B.Tourinho

\begin{abstract}
This paper discusses methods for developing diagnostic parameters for pedestrian traffic safety problems using the Traffic Conflict Analysis Technique (TCT), that were applied to a study of pedestrian crossings at urban signalized intersections in São Paulo, Brazil. The development of parameters has two tasks: the selection of an adequate classification of conflicts and/or segmentation of crossings and the determination of parameters for the classes (including recommended confidence levels). Based on the U.S.FHWA-Federal Highway Administration guides for study of vehicular conflicts, the pedestrian conflict count limits $C$ (abnormally high level of counts for problem detection) and the ratio $R$ of accidents per million of conflicts (risk index and accident forecasting rate) were developed for the types of urban crossings observed in the signalized intersections of the study. Usual statistical methods were applied with a new decision based criterion that could select the best classification/segmentation and the recommended confidence level to be used in applied work. Results obtained from the application of such methods to the case study are presented and discussed as an example application.
\end{abstract}




\section{INTRODUCTION}

This paper presents the discussion of methods used in determining diagnostic parameters for studies using the Traffic Conflict Analysis Technique (abbreviated here as TCT) and their application to the analysis of pedestrian safety at crossings of signalized intersections. The concepts and methods applied here to the TCT can also be used for other techniques.

Despite of failing to achieve widespread application, the need of evaluation methods based on direct observation of traffic operations, as the TCT, is a recurrent subject in academic and also in professional studies. Even after noting the effort in last years on developing practical safety performance models based on accidents, by themselves, a method of evaluation that use the direct observation of traffic operations is yet valuable for the safety analysis, by adding relevant information and responding in shorter time.

Due to a large international research effort carried-out during the eighties, the initial concept of traffic conflict was scientifically stated as an event in which two road users (or a road user and another traffic element) became in a course of collision and an evasive action is observed (braking, swerving or accelerating) to avoid the potential accident (see [1] for a historic background and the results of several studies of the international "calibration" effort).

After the joint effort, leading institutions of several countries published guides for applying TCTs, including recommendations and criteria for its use in the diagnosis of road safety problems (e.g. $[2,3,4,5,6])$ that are referred here as official guides. 
Among the procedures of these guides, one can distinguishes two ways in which the diagnosis using traffic conflict data can be carried-out.

The basic diagnosis, reported in all TCT guides, is based on the interpretation of traffic count data, relating them to traffic and site features and to the qualitative observations made during field observation. The classification of traffic conflicts by type and severity aids the analysis but the task of identifying a set of relevant safety problems (and of selecting proposals for improvement of safety) remains an expert challenge, as in the process of diagnosis based on accident analysis (more abundant and confident data is an advantage of TCT but this feature should be weighted against the risk of missing important factors in accident causation).

There is another type of diagnosis, recommended in the U.S.FHWA guide only (see [5]), that tries to identify types of traffic conflicts displaying abnormally high frequency compared to normal levels of traffic conflict counts (on a standard 11 hour period of a workday) and also tries to weight their accident proneness measured by the ratio of accidents to conflicts counts (a measure of the risk level of each conflict type that can also be used to forecast the expected accident frequency based on conflict counts). These refined diagnostic tasks take into consideration the type of conflict and the type of site (representing its physical and traffic features) and are based on objective diagnostic parameters.

One should note that the viability of the refined diagnosis is constrained by the availability of previously calibrated parameters on the abnormal level of counts and on the accident risk ratio of traffic conflicts, by conflict type and type of site, developed in a careful and representative 
study of a set of similar sites. The U.S.FHWA guide was based on data from such kind of previous studies $[7,8]$ for some usual classes of unsignalized and signalized intersections, but there was little progress in developing new data since then.

Despite this practical constraint, it is very important to stress the content of the refined diagnosis, underlining its meaning and relevance for the traffic safety analysis.

For example, a validation study $[9,10]$ counted the same-direction and transversal conflicts between vehicles in an unsignalized intersection with medium traffic level. The expanded count for the standard period was 239.0 same-direction and 133.5 transversal traffic conflicts. Nevertheless, based on the U.S.FHWA data, the corresponding limits for abnormal frequency are 410 and 24 (for $90 \%$ confidence level), saying that the less frequent conflict type is the more severe safety problem at the site. Also, again based on U.S.FHWA data, the accident/conflict ratio indicates that only transversal conflicts have a significant risk of generating accidents at this kind of site and, using the given ratio, it is possible to forecast a frequency of 13.6 collisions per year on workdays with dry weather. The record in the previous year was 16 (all accidents), every of them being transversal collisions, as predicted.

This real case study clearly shows the importance of the diagnostic parameters in the analysis.

From this setting, we built on our previous research $[11,12]$ and on the guidelines for TCT applied to vehicular conflicts (mainly in [5]) and searched for the development of diagnostic parameters for pedestrian-vehicle conflicts. Our main criteria in recording vehicle-pedestrian 
conflicts and our main results were previously reported [13]. Here our focus is on the method of study and, mainly, the new proposed criterion for selecting diagnostic parameters.

In the following, we try to present the usual approach used for determining diagnostic parameters for the U.S.FHWA TCT in section 2, including the proposal of a new decision based criterion that can be used for selecting the segmentation of crossings, classification of conflicts types and confidence level to be used in practical applications. The use of the methods in the case study and the results gathered are presented in section 3 , analyzing the selection of the classification of conflicts, the segmentation of crossings and the confidence levels. The final section advances some conclusions and suggestions for further research. 


\section{METHODS FOR DETERMINING AND EVALUATING DIAGNOSTIC PARAMETERS FOR PEDESTRIAN-VEHICLE CONFLICT STUDIES}

The diagnostic parameters used in the refined procedure associated to the U.S.FHWA TCT are based on observations of pedestrian-vehicle conflicts by type of conflict and type of crossing. The development of diagnostic parameters has two tasks: the selection of an adequate classification of conflicts and/or segmentation of crossings and for the estimation of parameter values for the selected classes (including confidence levels when required).

The procedure for determining the limit counts $\mathrm{C}$ for traffic conflicts recommended in the U.S.FHWA guide ([5], see also [8]) can be applied without major difficulties, after gathering the required data (conflict counts, expanded for the standard period). In this method, the abnormal level of the frequency of conflicts is determined through the analysis of the distribution of daily conflict counts per standard period, for each type of conflict, on similar sites of a class (procedures for collecting conflict counts and expanding the data to a standard period are simple and are also suggested in the U.S.FHWA guide [5]). Counts are usually obtained from short period observation (around 30 minutes each) and expanded for the standard period, filling the periods without counts based on an average rate (for workdays, the standard period of eleven hours recommended runs from 07 a.m. to 06 p.m.).

The procedure for determining the ratio $\mathrm{R}$ of accidents per conflict, or million of conflicts, is not described in the U.S.FHWA guide or related works (i.e. $[5,7,8]$ ). Nevertheless, the diagnostic parameter is a ratio of two random variables and available methods for ratio 
estimators are widely discussed in statistical textbooks on sampling theory (e.g. [14]). For achieving this task, traffic accident data has to be gathered and referred to the same observational units (intersections, approaches or crossings) and traffic conflict data has to be expanded to the same time frame of reference (e.g. workdays of a year). The procedures for expanding conflict counts for a year are presented in the U.S.FHWA guide ([5], in chapter 8, when discussing the forecasting of accident frequency using conflict data) can be used in this task of converting daily counts to yearly totals.

The evaluation of the diagnostic parameters should also be based on statistical criteria. There are conventional and simple recommendations on this subject but a decision criterion that is more adequate for engineering work seems to be preferable and is used in this work.

For completeness, the estimation procedures are shortly described in the following topic.

\subsection{Statistical Methods for Determining Diagnostic Parameters for TCTs}

The determination of diagnostic parameters for TCT has two peculiar features:

- basic variables have non-gaussian distributions and

- the risk measure is a ratio of random variables.

For establishing count limits $\mathrm{C}$, raw data are the expanded daily conflict counts for a standard period (e.g. the 11 hour period ranging from 7 a.m. to 6 p.m, for workdays, suggested in the U.S.FHWA guides). Accepting the empirical evidence that expanded daily conflict counts have a Gamma distribution ([14] apud [8]), their statistical parameters (s,t) can be estimated 
using some statistical method. For any statistical confidence level $\mathrm{L} \%$, count limits $\mathrm{C}_{\mathrm{L} \%}$ can be obtained accordingly using the parameters of the fitted distribution for similar sites and each type of conflict, as sketched in Figure 1.

$\langle$ Insert Figure 1>

Following the U.S.FHWA guide (see [5] and also [7, 8]), given a sample of sites, the estimates of the parameters using the method of moments are

$$
\begin{aligned}
& \mathrm{t}=\mathrm{m}_{\mathrm{C}} / \mathrm{s}_{\mathrm{C}}^{2} \\
& \mathrm{~s}=\mathrm{t} \cdot \mathrm{m}_{\mathrm{C}}
\end{aligned}
$$

with the average $\mathrm{m}_{\mathrm{C}}$ and the variance $\mathrm{s}_{\mathrm{C}}^{2}$ of daily counts, expanded to the standard period.

The count limits $\mathrm{C}_{\mathrm{L} \%}$ for similar sites and each type of conflict, for the selected statistical confidence levels L\%, can be determined using Chi-square tables and the relationship between Chi-square and Gamma random variables (see [5], pp.62-63). Knowing that the critical value of the Gamma distribution (the count limit) is given by $\mathrm{C}_{\mathrm{L} \%}=\frac{\mathrm{X}_{\mathrm{v}, \mathrm{L} \%}^{2}}{2 . \mathrm{t}}$, where $\mathrm{X}_{v, \mathrm{~L} \%}^{2}$ is the critical value of the Chi-square distribution with $v=2 . s$ degrees of freedom corresponding to the same L\% confidence level (or percentile), calculations can be carried-out with a standard Chi-square table, for selected values of $\mathrm{L} \%$. Given the parameters of the 
fitted distribution and applying the formulas above, one has to calculate $v$ and interpolate for the value of $\mathrm{X}_{\mathrm{v}, \mathrm{L} \%}^{2}$ in a Chi-square table, before the count limits $\mathrm{C}_{\mathrm{L} \%}$ are calculated.

Nowadays, all calculations can be carried-out directly with simple spreadsheet software (as example, MS-Excel has the functions GAMMADIST and GAMMAINV for the density/cumulative gamma distribution and its inverse cumulative probability function, using the more usual description of the Gamma distribution with parameters $a=s$ and $b=1 / t$ ).

There is no suggested procedure for the statistical evaluation of the estimated count limits or their performance in identifying unsafe sites in the U.S.FHWA guide [5]. A conventional option could evaluate the fitting of the statistical distribution with the Kolmogorov-Smirnoff goodness of fit measure D. Using the previously quoted statistical properties and being $\mathrm{i}$ the index of each count $\mathrm{C}_{\mathrm{i}}$ in increasing order of count value, it is easy to verify that the sample value of the Kolmogorov-Smirnoff statistic is $D=\max _{i}\left\{\left|\frac{i}{n}-F_{C_{i}}\right|,\left|F_{C_{i}}-\frac{i-1}{n}\right|\right\}$, where $\mathrm{F}_{\mathrm{C}_{\mathrm{i}}}$ is the cumulative probability at $\mathrm{X}_{\mathrm{v}, \mathrm{i}}^{2}=2 . \mathrm{t} . \mathrm{C}_{\mathrm{i}}$ for $\mathrm{v}=2 \mathrm{~s}$ in the standard Chi-square table.

Nevertheless, a decision based criterion for analyzing the performance of the count limits, selecting the best classification of conflict types and/or segmentation of sites and also the best confidence level for practical applications is suggested in the section 2.2 ahead.

For establishing the risk measure $\mathrm{R}$, and its variance, several conventional methods of determining ratio estimators of sampling studies can be used. The most common estimators 
are the ratio of means (or totals) and the mean of ratios. Both are biased estimators and Cochran (see [15], chapter 2 or 6) suggests the use of the ratio of means (or totals) as it delivers a smaller mean square error (where $E[R]^{2}=\operatorname{Bias}[R]^{2}+\operatorname{Var}[R]$, with $\operatorname{Bias}[R]$ the expected bias of the estimator and $\operatorname{Var}[\mathrm{R}]$ its variance). The analysis considers all sites of the same segment or a pool of segments, for each type of conflict or some aggregate type.

For a class, with the total number of accidents $\left(t_{A}\right)$ and conflicts $\left(t_{C}\right)$ for all the sites in the sample or the average values of accidents $\left(\mathrm{m}_{\mathrm{A}}\right)$ and conflicts $\left(\mathrm{m}_{\mathrm{C}}\right)$ in the sample of sites, the estimators of the ratio $\mathrm{R}$ and its standard deviation are:

$$
\begin{aligned}
& \hat{\mathrm{R}}=\frac{\mathrm{t}_{\mathrm{A}}}{\mathrm{t}_{\mathrm{C}}^{\mathrm{E}}}=\frac{\sum \mathrm{A}_{\mathrm{i}}}{\sum \mathrm{C}_{\mathrm{i}}^{\mathrm{E}}}=\frac{\mathrm{m}_{\mathrm{A}}}{\mathrm{m}_{\mathrm{C}}} \\
& \mathrm{s}(\hat{\mathrm{R}})=\frac{1}{\mathrm{~m}_{\mathrm{C}} \cdot \sqrt{\mathrm{n}}} \cdot \sqrt{\frac{\sum\left(\mathrm{A}_{\mathrm{i}}\right)^{2}-2 \cdot \hat{\mathrm{R}} \cdot \sum\left(\mathrm{A}_{\mathrm{i}} \cdot \mathrm{C}_{\mathrm{i}}^{\mathrm{E}}\right)+\hat{\mathrm{R}}^{2} \cdot \sum\left(\mathrm{C}_{\mathrm{i}}^{\mathrm{E}}\right)^{2}}{\mathrm{n}-1}}
\end{aligned}
$$

where $\mathrm{A}_{\mathrm{i}}$ and $\mathrm{C}_{\mathrm{i}}^{\mathrm{E}}$ are the expanded frequencies of accidents and conflicts for each site and $n$ is size of the sample of sites for a given class (see [15], pp.30-34).

The expansion of conflict data to totals per year are carried-out using notional conversion factors (instead of using methods based on statistical distributions or empirical coefficients) as in the procedure recommended by the U.S.FHWA guide ([5]). The advantage of using notional conversion factors is that they do not contribute to inflating parameter variance. The disadvantage is a potential reduction in the precision of the risk measure. 
However, note that the decision of using the same time period for accident and conflict data is not mandatory as long as they refer to the same type of period. The option of relating data obtained from the same time frame but using different unit of time is a viable one (that would avoid complex extrapolations). These parameters are called rough ratios (RR, e.g. in yearly accidents per hourly conflicts) and are best suited for practical purposes (even if less precise).

For both ratios, the statistical quality of the estimate is measured by its standard deviation, its variance or, relatively, by its coefficient of variation (the inverse of a " $\mathrm{t}$ " statistic). Tests on the difference between ratios, with the assumption of independent samples, can be carried-out only approximately (as the distribution of the ratio estimators is non-normal and unknown), based on a standard t test of the quasi-t statistic (see [15], pp.180-183). Then, the comparison of the difference of ratios on two disjoint segments of crossings can be carried-out (at least approximately) and its statistical significance asserted as long as the factors that generate dependence between counts on the sites of each segment are of minor importance.

The practical performance of the ratios as estimators in forecasting accident frequency can also be analyzed by evaluating the expected error of estimates, following the procedures recommended in the U.S.FHWA guide [5]. However, the comparison of predicted and observed accident counts (or yearly frequencies) on a sample of sites is also possible, as long as the sample is not subjected to usual selectivity biases or regression to the mean effects is accounted for. This option is more revealing by displaying the explained and unexplained variation of the conflict ratio forecasting method and was carried-out in the application. An external validation study (with other sample) would do better, evaluating transferability also. 
The option of comparing the forecasting errors achieved (expected or observed) can evaluate several different methods (e.g. accident to conflict ratio, accident to exposure ratio, accident regression models, empirical Bayesian procedures). Nevertheless, it is important to weight the content of each method and the way it is applied in studies. For example, the TCT method is almost unique in using observational tips from the traffic operation (a competitor would use, at most, basic variables as traffic volume or speed) and can be applied immediately (so does not have to wait for accidents to happen). The error is just one of the things that matter.

\subsection{A Method for Evaluating Segmentations and Confidence Levels for Diagnostic Parameters of TCTs with a Decision based Criterion}

Instead of searching for purely statistical criteria, the performance of alternative segmentation or confidence levels can be evaluated using a loss function based on the benefits and costs of implied decisions that will be motivated by the use of the parameters. The same approach can be applied to the other settings as well, with small methodological changes.

For TCTs, these decisions correspond to the selection of sites for treatment. The costs and benefits are related to:

- the realization of the potential accident reduction with treatment of risky sites;

- the waste of resources devoted to safe sites also selected for treatment,

- the saving of resources of avoiding the treatment of safe sites

- the remaining social costs of accidents for risky sites not selected for treatment. 
Each effect must be evaluated given parameters used in the diagnostic activity that select sites for treatment and data available on the partial effectiveness of treatments (taking into consideration the persistence of unavoidable accidents with usual countermeasures).

An internal validation study can be carried-out applying this analysis to the sample used for the establishment of parameters. An external (or cross) validation study would conduct this exercise in another sample of sites and will evaluate the transferability of the parameters as well. The evaluation can be extended to the monitoring of costs and benefits of the treatments or can use average or representative measures of potential costs and benefits.

Using this terminology, the application that will be reported in this study will do an internal validation with representative measures of costs and benefits.

Our procedure supposes that the TCT is used in selecting sites for treatment by comparing average daily counts (for any number of days) to the count limits of a given confidence level, then deciding to treat the sites with abnormal frequency of conflicts of any type. The limit counts can be developed with alternative segmentation proposals (even aggregate).

The procedure also supposes that all selected sites are treated and that representative values of costs and benefits are available for the four possible cases, as depicted in Table 1. We prefer to communicate benefits and costs in US\$ (generating a kind of economic weighted index) but, of course, any other agreed compensatory scale can be used (even non-monetary). The values shown are representative of direct or material costs of accidents with average severity 
in Brazil and were selected for illustrative purposes on conservative grounds (other criteria, including human capital and/or pain and suffering components, could be used as well).

\section{$<$ Insert Table 1>}

With economic based weights, the overall evaluation criterion is a kind of net economic index, following usual criteria applied in Benefit/Cost Analysis. Using the hypothetical representative weights suggested in Table 1, the net economic index E can be calculated with data on the number of sites for each represented cell (NEI, NEII, NHI, NHII) and the corresponding number of accidents (AEI, AHI only on error type I and hit type I sites). The number of sites with accident is $\mathrm{NA}=\mathrm{NHI}+\mathrm{NEI}$, the number of sites without accidents is $\mathrm{NWA}=\mathrm{NHII}+\mathrm{NEII}$, the number of treated sites is NTS $=\mathrm{NHI}+\mathrm{NEII}$, the number of avoided accidents is $\mathrm{AA}=\mathrm{AHI}$ and the number of remaining accidents is $\mathrm{AR}=\mathrm{AEI}$.

Admitting that accidents are only partially avoidable through engineering treatments, the costs of unavoidable accidents can be ignored. Nevertheless, the treatment costs must be computed on all sites selected for treatment. A restricted net economic index would evaluate benefits at treatment sites only (ignoring the remaining accidents on non-treated sites with accident records) but a better measure of net economic performance can be constructed noting that the "do nothing" scenario has a large negative net economic index that should be corrected.

With weights given by $\alpha_{\mathrm{S}}$ (potential accident savings) and $\beta_{\mathrm{C}}$ (treatment costs) as defined in Table 1, the net economic index of avoidable costs in the "do nothing" (E0) option is 


$$
\mathrm{E} 0=-\alpha_{\mathrm{S}} \cdot \mathrm{A}=-\alpha_{\mathrm{S}} \cdot(\mathrm{AHI}+\mathrm{AEI})
$$

and the net economic index attained with treatments selected based on a given criterion is

$$
\mathrm{E}=-\beta_{\mathrm{C}} \cdot \mathrm{NTS}-\alpha_{\mathrm{S}} \cdot \mathrm{AR}=-\beta_{\mathrm{C}} \cdot(\mathrm{NHI}+\mathrm{NEII})-\alpha_{\mathrm{S}} \cdot \mathrm{AEI}
$$

(that can be better than the "do nothing" measure even if negative).

Based on conventional Benefit/Cost Analysis, a differential measure of the net economic index against avoidable costs in the "do nothing" scenario (DE) should be built as

$$
\mathrm{DE}=\mathrm{E}-\mathrm{E} 0=\alpha_{\mathrm{S}} \cdot \mathrm{AHI}-\beta_{\mathrm{C}} \cdot \mathrm{NHI}-\beta_{\mathrm{C}} \cdot \mathrm{NEII}
$$

that is the usual restricted measure of economic performance based on treated sites only (as the number of avoidable accidents on non-treated sites cancel out).

The maximum attainable net economic index of treatment options (ME) is

$$
\mathrm{ME}=\alpha_{\mathrm{S}} \cdot \mathrm{A}-\beta_{\mathrm{C}} \cdot \mathrm{NA}=\alpha_{\mathrm{S}} \cdot(\mathrm{AHI}+\mathrm{AEI})-\beta_{\mathrm{C}} \cdot(\mathrm{NHI}-\mathrm{NEI})
$$

(delivering ) and a relative measure of performance over the maximum attainable (RE) is

$$
\mathrm{RE}=\frac{\mathrm{DE}}{\mathrm{ME}}=\frac{\alpha_{\mathrm{S}} \cdot \mathrm{AHI}-\beta_{\mathrm{C}} \cdot \mathrm{NHI}-\beta_{\mathrm{C}} \cdot \mathrm{NEII}}{\alpha_{\mathrm{S}} \cdot(\mathrm{AHI}+\mathrm{AEI})-\beta_{\mathrm{C}} \cdot(\mathrm{NHI}+\mathrm{NEI})}
$$


that is the improved economic measure previously quoted (considering all observed accidents in the validation sample).

The best segmentation and confidence level for decision is the one that delivers the greater value of the performance measure, provided that its net economic index is better than the "do nothing" option. Even if not a perfect option (approaching 100\% of ME) or if not the best possible option, any criterion with $\mathrm{E}>\mathrm{E} 0$ (or $\mathrm{DE}>0$ ) is defendable. The worst possible result is $\mathrm{WE}=-\beta_{\mathrm{C}}$.NWA but any policy delivering $\mathrm{DE}<0$ is worse than the "do nothing". The performance measures need not deliver a positive net economic index per se (as the status quo carries significant social costs) but the treatments should attain usual positive net economic return, represented by the differential net economic index DE (preferably with a large ME).

These set of measures also have other very convenient properties:

- The differential measure DE, being a linear combination of results on the number of sites and/or accidents in each outcome cell, delivers the overall achievement of any segmentation as the summation of the DE on each segment, revealing where the best or worst contributions to the overall results were gathered. The segments where the DE partial achievements have poor performance are natural candidates for redefinition or further segmentation.

- The relative measure RE, being bounded by $100 \%$, has a clear meaning for measuring the final performance against a tight limit. Any criterion with positive DE is usable, based on economic ground, but criteria that can reach RE measures of $80 \%$ or $90 \%$ are 
also at the limit of the possibilities for whatever criteria (at least for those calculated based on the same set of established parameter values).

This evaluation approach can not be used directly to the validation of the ratio $\mathrm{R}$ of accidents to conflicts as there is no clear decision related to its use in the conventional approach.

The previously quoted options of evaluating the statistical significance of differences in $\mathrm{R}$ for alternative classification/segmentation (using the quasi-t statistic with the standard t test as an approximation) and of evaluating the accident estimates obtained with the accident to conflicts ration against observed accident frequencies, both were used in the analysis of parameters values. Nevertheless, in the comparison with observed accident frequencies, the technical requirement of using individual pedestrian crossings as units of analysis (instead of intersections) is a problem, given the small number of accidents in each observation.

Before leaving the theoretical discussion, two additional comments are worth of notice.

First, note that a decision criterion to select sites for treatment based on the accident estimates obtained from conflict counts can be proposed (being practical or artificial, i.e. that will be used for evaluation purposes only). Then, ratios would be amenable to the same decision based method of evaluation applied for the classification/segmentation and confidence level of count limits. The criterion could be based on the expected number of accidents predicted (e.g. greater than 1 in a year) or on some figure derived from an approximate distribution of the estimate (e.g. more than $75 \%$ chance of a pedestrian accident in a year). In each case, the selection of value for the criterion could be also carried-out with the proposed method. 
Second, note that the proposed method is reminiscent on the view of traffic conflicts as proxy to traffic accidents (taken as the "true" measure of unsafety, as the only one that should be considered and whose reduction would be weighted as benefit of the actions). A wider view considers a traffic conflict as a safety measure per se and as an event that brings discomfort to road users even if the danger of accident is avoided (as any other related traffic event that burdens on safety). Then, one can estimate the value to road users of reducing traffic conflicts (i.e. the "cost" of traffic conflicts, similarly to the "cost" of accidents, travel time and the like). Its inclusion in the net economic indexes is fully justified based on a Benefit/Cost rationale, at least as long as it represents objective discomfort. However, as a measure of accident risk, its role is played in the forecasting of accident frequencies (and double counting should, then, be avoided). This same reasoning would suggest that other effects of safety measures (as the increase in travel time brought by a reduction of speed mandated by safety goals) should also be included as well. These points are limitations that could ask for improvements when evaluating other techniques or some specific countermeasures.

In the case study, representative values were used and a sensitivity analysis carried-out for most parameters values, as reported in the following section, but always keeping the initial approach described here of weighting only the benefits of accident reduction. 


\section{APPLICATION TO THE DETERMINATION OF DIAGNOSTIC PARAMETERS FOR PEDESTRIAN-VEHICLE CONFLICTS IN THE SÃO PAULO STUDY}

The diagnostic parameters for pedestrian-vehicle conflicts were obtained based on the 1998 study carried-out in 26 pedestrian crossings of four critical signalized intersections of the City of São Paulo (see [12]).

The main interest here is on the description of the application of the decision based criterion and the way it fills the role of supporting the development of parameters, mainly the selection of segmentation and confidence level. The study will be summarized for the interested reader in section 3.1, that can be skipped without loss of continuity. The main analysis is carried-out in section 3.2 that also present the main results (which were previously reported in [13] also).

Conflict types were based on a preliminary classification shown in Figure 2.

<Insert Figure 2>

Table 2 has a simplified sketch of each intersection and shows the position of each pedestrian crossing (using its number in the intersection). The abbreviated notation used for identifying crossings is cumbersome but is also kept here for precision.

<Insert Table 2> 
The intersections are identified in the corresponding column of Table 2. Two intersections are located in the old central area (Ip-SJ and Co-CP). The other two are located in southwest of the expanded central area (FL-TS and FM-VB).

Pedestrian crossings were classified and nominated as done in the original study [12], as identified in the corresponding column of Table 2.

Common features of all signalized intersections and their crossings are:

- high vehicular and pedestrian flows, with averages around 1.500 vehicles and 1.000 pedestrians per hour in each crossing;

- $\quad$ road markings (mainly painted crossings and stop lines) in good conditions, where provided, but significant pedestrian movements were observed also in some unmarked crossings;

- the flows related to commercial activities and transit services is very important in all the areas;

- carriageways have at least two lanes and are one-way (two-way roads have separated carriageways with raised medians);

- there are signal groups for vehicle and pedestrians at all intersections but one (the FLTS intersection);

- especial lanes and especial phases for turning vehicles are absent and left turn are locally forbidden and rerouted through loops on adjacent streets. 
Vehicle and pedestrian flows are shown in Table 2. Traffic operations at the sites were registered with video-cameras in the morning, mid-day and afternoon peaks ( 2 hours in each period), along with the counting of conflicts by field personnel. Due to a failure in the positioning of the video-camera at one site, recording of pedestrian flows were missed on one of its crossings and the corresponding data had to be discarded from the analysis.

Finally, accident data from police records are summarized in Table 3. Note that only part of the registered accidents had the official police accident reports recovered.

<Insert Table 3>

\subsection{Data Gathered at the Studied Crossings}

As previously mentioned, two sources of data were used in the study:

- data on pedestrian accidents, used for the determination of accident to (million) conflicts ratios and as the validation criterion on the detection of unsafe sites based on conflict counts;

- data on pedestrian-vehicle conflicts, used for the determination of the count limits for detecting sites with abnormal level of conflicts and as the diagnostic tool on the detection of unsafe sites based on conflict counts.

Details on the gathering and processing of data are shortly described in the following topics. 


\section{a. Data on Pedestrian Accidents at the Studied Crossings}

Accident data were recovered from police records and police reports for two years before the conflict study (1996 and 1997). It was impossible to recover some of the accident reports of each intersection. Table 3 summarizes data for all the registered accidents and for recovered accident reports. Accidents referred to in Table 2 are only those with recovered reports and that occurred during the standard period of workdays (the same period of reference used as the standard period for expanded conflict counts).

As can be seen, 26 reports were recovered from 54 registered pedestrian accidents and, among them, only 13 from 29 on the standard period of workdays. The accidents with recovered police reports were used in all analysis, except when estimating ratios of accidents to conflicts, for which the total number of accidents in the standard period of workdays was adjusted by the ratio of the known total to the recovered total, as described in the following, knowing that 29 accidents, instead of 13 only, occurred in the standard period of workdays (other accidents are not included).

For each accident with a recovered accident report, an accident location index (\%) had to be defined due to the lack of precise data on some police reports (see the column corresponding to accidents in Table 2). Most accidents were clearly located (i.e. had a 100\% location index) but 6 of 13 pedestrian accidents had from 75/25 to 50/50 as accident location index (split between two crossings, usually adjacent and/or similar) because pedestrian and vehicle movements were not clearly identified in most police accident reports (except when there was an obvious pattern commanded by the intersection layout and/or crossing site). 
Nevertheless, it was possible to verify that there was sites with pedestrian accidents among near crossings with through flow (as 1:Co-TA-BC, 1:FM-TP-CB e 3:Ip-E-TP) and among far crossings with turn flows (as 2:FL-TA-IP e 7:MA-TA).

The accident location index was interpreted as the probability of occurrence of the accident in each crossing and the aggregate accident index was taken as the empirical estimate of the expected number of accidents in each crossing. The validation criterion for application of the TCT took the crossings with non-null accident index as unsafe and the others as safe (i.e. it is supposed that missing accidents occurred in the same crossings and that accident records were able to identify all the risky crossings). The suppositions described above would not be required when using better data (an unavoidable problem in our case study).

\section{b. Data on Pedestrian Conflicts at the Studied Crossings}

Conflict counts were carried-out during two days (a Monday and a Tuesday) of march, 1998 (actually, 16 and 17 of march), from 07:30 to 18:30 (using 6 one hour count and half hour rest periods, separated by one and a half hour for lunch just before or after mid-day, in each day, and amounting to 312 hours of observation on all crossings). Both days had good weather (and dry pavement). Recorded counts were expanded to the standard period of each day, following U.S.FHWA recommendations [5], and the average daily counts were obtained for each crossing and subsequently used in all analyses. 
Table 4 summarizes the data obtained for each crossing. One can note that sites with higher pedestrian-vehicle daily counts are not always the sites with pedestrian accidents (near crossings with through flow, as 1:Co-TA-BC, 1:FM-TP-CB e 3:Ip-E-TP, and far crossings with turn flows, as 2:FL-TA-IP e 7:MA-TA). This observation stresses the need of diagnostic parameters for selecting unsafe sites with better success. Table 4 also shows the main candidate variables for segmentation of crossing types (in column Seg.I and column Seg.II) as will discussed in the following. Conflict types were also similarly analyzed for aggregation.

$<$ Insert Table 4>

\subsection{Development of Parameters for the TCT Application to Pedestrian Safety Studies}

The development of parameters for the application of the TCT in the safety analysis with pedestrian-vehicle conflicts based on the U.S.FHWA guide includes:

- selecting a relevant segmentation of crossings;

- selecting a final classification of conflict types;

- determining limit counts for selected confidence levels;

- determining accident to (million) conflicts ratios.

For the development of limit counts, the decision based criterion is straightly applied as there is a clear recommendation of selecting sites for treatments using on the comparison of average field counts to the established count limits, with a given confidence level, for all the conflict types discriminated on the type of crossing of each site. The decision based criterion is used to select the best segmentation of crossings, classification of conflict types and confidence level. 
The net economic index had to be computed with the sample of recovered accidents. With economic weights suggested in Table 1 (i.e., a 50\% reduction potential with a unit cost of US\$ 20000 per accident and a treatment cost of US\$ 5000 per crossing), the actual "do nothing" or current situation can be associated to a loss of US\$130000 related to the potential direct cost reduction (50\%) for 13 accidents. The treatment costs amount to US\$ 60000 in all the 12 accident sites (and US\$ 70000 in all the 14 non-accident sites).

Then, the lower and upper thresholds against which the net economic index of alternative TCT detections should be compared are:

- full success ME with treatment of the 12 sites with accidents only and a net benefit of plus US\$70000 (savings of US\$ 130000 less the cost US\$ 60000 in 12 sites) and

- full failure WE with treatment of the 14 sites without accidents and a net benefit of minus US\$ 70000 (avoidable accident costs are US\$ 130000 as in the "do nothing").

Note that relative values (RE) against the maximum (ME of US\$ 70000) should be less sensible to benefit and cost weights for the comparison of alternative diagnostic criteria. Absolute values (DE) should be taken as conservative ones in the subsequent discussion.

For the development of accident to (million) of conflicts ratios, the conventional procedures for establishing parameters has to be used, as there is no recommended decision associated to the use of the risk measure. A clear comparison of the alternative procedures can be made. 


\section{a. Determination of Count Limits for Daily Conflicts}

Count limits $\mathrm{C}$ were determined for several segmentations, constrained by data availability. It is easy to see in Tables 2 and 3 that detailed segmentation of crossings is impossible with our data as the sample size in each segment quickly becomes too small and the validation analysis loses interest (as all the sites have no accident in the sample of some segments).

The raw data on conflict counts is used as summarized in Table 4.

The main segmentation criterion for the type of crossing distinguishes TP (i.e. near or stop line crossings) and TA (far or free crossings) sites. Remembering that all the carriageways are one-way, this is a fundamental classification based on pedestrian vehicle interaction and signs one version of the general distinction between protected and concurrent crossings.

On TP crossings of signalized intersections, conflicts can occur only on traffic violations by pedestrians or vehicles and this possibility increases sharply when there are available gaps on vehicular flow. Then, further segmentation was considered based on the level of saturation of the vehicle approach (TP-Sat or TP-NSat).

On TA crossings, there are concurrent movements of pedestrian and vehicles even without violations and conflicts can occur also in this situation. Then, segmentation based on pedestrian flow was analyzed because some crossings have very high traffic of pedestrians (TP-Ped or TP-Ped+, taking $900 \mathrm{ped} / \mathrm{h}$ as the threshold of high pedestrian flow crossings). 
In the following, the basic terminology used to classification of conflict types or segmentation of crossing types is: aggregate/disaggregate for pooled or distinct groups of conflict types and complete/segmented for pooled or distinct groups of crossing types.

Results are presented for the complete sample (with aggregate conflicts or disaggregate analyses by conflict type with 2,4 and 8 types) and also for the TA/TP segmentation (with aggregate conflicts or disaggregate analyses by conflict type with 2 and 4 types). The category of each crossing for the main segmentation can be seen checking the column Seg.I of Table 4. Both analyses are carried-out for three levels of confidence $(75 \%, 90 \%$ and $95 \%)$.

More refined segmentations, with TP-Sat/TP-NSat and TP-Ped/TP-Ped+ were also submitted to a preliminary study of aggregate and disaggregate analyses. The refined category of each crossing can be checked on the column Seg.II of Table 4. Despite the small samples available in each cell, the preliminary study of more detailed data can suggest the potential gain from further segmentation (and its effect on the need of a disaggregate analysis of conflict types).

Table 5 summarizes count limits based on the sample of crossings, taking several alternatives for segmentation of crossings and disaggregation of conflict types. Part 5a contains the basic data for the complete sample and the segmentation of crossings in two groups TA/TP, with 4 types (P/TP, P/TA, P/TD and P/TE), with 2 types (P/VA, with P/TP and T/TA, and P/VT, with P/TD and PTE) or pooling all conflicts in an aggregate type. Part 5b contains preliminary data on more detailed analysis with further segmentation (TA-Ped/TA-Ped+, TP-NSat/TPSat) or disaggregation (all the 8 conflict types of Figure 2). 
$<$ Insert Table 5>

Based on the count limits, the diagnosis of safe or unsafe site is carried-out for the crossings and compared to the accident records. Note that the use of the accident location index spreads accidents among crossings but acts in the opposite direction of using the diagnosis based on the sample of recovered police accident reports only. Alternative analyses with rounding of the cumulative accident index of each site delivered comparable results and the analysis based on a 50/50 split of accidents (i.e. with less precise location) were kept for presentation.

The main result of the paper, in Table 6, presents the evaluation index of each alternative segmentation and disaggregation and also for each of the 3 confidence levels. The indexes were evaluated using the representative weights of Table 1. The nomenclature for errors type I and II or hits type I and II are also the same of Table 1 as well.

$<$ Insert Table 6>

The lower confidence level was introduced based on the observation that when higher accident savings are conjectured the gain achieved is very significant (i.e. a more stringent statistical criterion runs against safety). Based on our data, the use of the $75 \%$ confidence level with the TA/TP segmentation and aggregate conflicts is clearly preferred (the TA/TP segmentation and the classification with 4 or 8 conflict types is also recommended).

The compromise between Type I and Type II hits (or errors) is clearly shown in Table 6 when comparing the $\% \mathrm{Hit} 1$ and $\% \mathrm{Hit} 2$ (the preferred alternative reaches $41.67 \%$ hits on identifying 
accident sites and $71.43 \%$ hits on identifying safe sites). The effect of requiring higher confidence levels is clearly displayed (increase of \%Hit2, identifying safe sites, at the expense of decreasing \%Hit1, identifying unsafe sites). Note that the TP/TA segmentation is firmly supported for all disaggregation of conflict types, being essential for achieving good results when using up 2 conflict types. The TP/TA segmentation is implicit in the use of 4 or 8 types but the interesting point here is that the use of more refined conflict types can change the pattern of errors by favoring safety. For example, with the $75 \%$ confidence level and the TA/TP segmentation, the use of 8 conflict types increases $\% \mathrm{Hit} 1$ to $58.33 \%$ (detection of unsafe sites) and decreases $\%$ Hit2 to $64.29 \%$ (detection of safe sites).

The absolute or relative economic indexes are handsome in delivering an unambiguous selection. Note that $\mathrm{DE}>0$ is achieved in all cases when using the $75 \%$ confidence level with the TA/TP segmentation or at least 4 conflict types. The use of the TA/TP segmentation (again implicit in the use of 4 or 8 conflict types) is clearly superior. Nevertheless, the relative index shows clearly that more insightful classification/segmentation is needed.

For getting clues on improvements, it is interesting to note that the DE can be (additively) decomposed in each sub-sample of crossing types. One can see that the net economic performance is worse in crossings of the TA-Ped and TP-NSat groups (suggesting where better data and segmentation would perhaps bring some additional precision in these classes).

Some results not shown in Table 6 are also worth noting. During the evaluation, it became clear that the result on the option with the best performance index was sensible to the values of weight for most confidence levels. With representative values at the border of economic 
efficiency for treatments (an accident saving of US\$ 7500 with the treatment cost of US\$ 5000), the same pattern of results was obtained. Higher accident saving values will favor even more liberal criteria, on behalf of safety benefits. Then, the use of more stringent statistical criteria (as the 95\% confidence level included in the U.S.FHWA guide [5]) seems to be overly conservative on economic grounds in any reasonable context.

Note that the use of the more detailed segmentation is promising, based on our results. The same pattern seems to be suggested by results from the more detailed disaggregation of conflict types but, in this case, better performance is limited to higher accident savings values, especially for higher confidence levels, what seems to be due to the selection of a larger number of sites for treatment (nevertheless, the same can be more conveniently achieved by lowering the required confidence level).

\section{b. Determination of Ratios of Accidents per Million Conflicts.}

The difficulty in identifying movements of vehicles and pedestrians involved in traffic accidents commanded an aggregate analysis of the risk measure based on the ratio of accidents per million conflicts. The following analysis is, then, limited to the comparison of the ratio on each crossing type (all, TA/TP, TA-Ped/TA-Ped+ and TP-NSat/TP-Sat).

The conversion of daily conflicts in the standard period to annual conflict totals is carried-out using representative factors as displayed in Table 7 (suggested in [5] and recommended in [9] also). As our results are limited to workdays and dry pavements, the ratios can also be 
calculated as a rough ratio RR of yearly accidents per hourly conflict. Despite its simplicity, we applied this procedure previously with good practical results.

$<$ Insert Table 7>

With such conversion factors, the calculation of the annual frequency of traffic conflicts is straightforward and can be related to the annual frequency of accidents (given our two-year data samples). Nevertheless, as our sample of accidents was only able to recover data on 13 police reports of 29 occurrences, we had to eliminate this partial data bias by inflating the estimated ratio by a correcting factor $(29 / 13=2.23)$. One should note that this correction does not account for partial record of occurrences (that would also be present in our data as usual).

Table 8 summarizes the results on the ratio of accidents per million conflicts for each of the pedestrian crossing segments used in this study. Part 8a shows that the difference of the ratio of the risk measure of conflicts in TA and TP crossings is suggested to be highly relevant (1 to 8 or one order of magnitude) and also statistically significant (at least based on the approximate test on the quasi-t statistic). A more detailed analysis is shown on Part $8 \mathrm{~b}$. At a smaller degree, the same confidence can be attributed to the difference between the risk measures of conflicts on TP-NSat and TP-Sat crossings and clearly point to the importance of diagnostic parameters for a proper analysis. Despite the high variance, only the segmentation of TA crossings based on pedestrian flows is discarded as non-relevant and non-significant. This conclusion can be attributed to the high level of pedestrian flow (900 ped/h) used as a threshold between classes. As our sample have a very small number of crossings with small pedestrian flows, our choice was constrained. 
One can clearly see that differences even when the values of the ratio are relevant based on an engineering criteria but the statistical significance is reduced by the high variance of the estimates. Use of empirical conversion factors in the expansion of daily conflict into yearly million of conflicts could improve the forecasting of accidents but also could increase the variance of estimates (15, chapter 8$)$.

For example, the difference of the ratio on TP-NSat and TP-Sat crossing types is relevant yet (almost 1 to 4), despite less significant statistically. The comparison of these two segments of crossing types is also illustrative of the meaning of the diagnostic parameters. Note that the risk measure of conflicts is higher in crossings of the TP-Sat type (in stop lines of saturated vehicular approaches). Nevertheless, the frequency of conflicts is higher in crossings of the TP-NSat type (almost 2 to 1 , as shown by the average count in Table $5 \mathrm{~b}$ ). Then, crossings of the TP/NSat type were predicted as finally safer (taking the product of each measure).

A last point can be made about the precision of estimates of the expected accident level based on the use of the accident to (million) of conflict ratio. As previously mentioned, the comparison with predicted and observed counts can reveal the explained and unexplained error achieved with different methods and should be weight against the specific content of each method (as the safety diagnostic based on the direct observation of traffic operations that the TCT is able to carried-out but can not be achieved by most alternative methods). 
Just for accident prediction, the different methods can be assessed as competitors and, as an example, be embodied into an Empirical Bayesian procedure (the currently preferred statistical evaluation method in safety research). In this case, the prior expected accident frequency can be estimated from accident counts on sample of similar sites, from exposure or conflict based ratios or from statistically calibrated model equations (a more sophisticated technique that can use several explanatory variables, that in the other techniques are usually considered only in developing segmentation alternatives). Despite originated by the need to take into account the regression to the mean effect (see [16]), the EB approach can be used as a variance reduction tool with all the other methods.

Table 8 includes data from the absolute average and maximum errors achieved with accident prediction on the samples based on the use of the ratio estimators and the accident counts on each the crossing segmentation considered. With accident counts, the average accident frequency in each segment was used as predictor (corresponding to the prediction based on the sample mean). Based on their estimating equations (see [17], item 3.2), it can be shown that the same predictors are generated by a poissonian or negative binomial regression model for accident frequency with group specific dummies as explanatory variables.

As can be seen, average and maximum absolute errors are large and similar for both methods but more dependent on the segmentation for the ratio estimates (at least without conflict types). Then, more sophisticated models/refined segmentations is a need but the precision achieved with the ratios is comparable to similar simple models based on accidents. 


\section{CONCLUSIONS}

This research aimed at analyzing methods used in determining the diagnostic parameters for the analysis of pedestrian safety problems at signalized intersections based on the traffic conflict analysis technique (TCT), using the U.S.FHWA concepts for refined diagnosis.

The methods used for the determination of diagnostic parameters were reviewed and a new decision based criterion for tracking the practical performance of their use in applied work was proposed as a complement to conventional statistical criteria. Both approaches were applied to a case study using a sample of crossings with high vehicular and pedestrian flows of the City of São Paulo, Brazil, for illustrative purposes.

For establishing limit counts, the parameters used as comparator in selecting sites for treatment based on their field data, the proposed method was shown to be able to select the best segmentation and confidence levels for count limits to be used in practical work. The criterion has a clear economic interpretation and can be decomposed additively so as to reveal sub-samples where achievements are better or worse.

The results on the criterion for identifying abnormal conflict counts recommended the use of a $75 \%$ confidence level and at least the segmentation of near or stop-line crossings (TP) and far or free crossings (TA). The use of 4 conflict types (the same types used in the U.S.FHWA guide) or 8 conflict types are also suggested on a preliminary analysis but more data is need to confirm that further benefits can be expected from more detailed parameters. Nevertheless, 
the study showed that the decision on the best criteria is sensitive to benefit and cost parameters, what can constraint the scope for detailing.

For accident to (million of) conflicts ratio, conventional statistics were used for the same alternative groups of crossings as there is no clear decision implied by the ratios or by the accident predictions based on their use.

For the measure of risk (accident proneness), constrained by the impossibility of a precise identification of vehicle and pedestrian movements involved in accidents, a relevant and significant difference between the ratio of accidents per million conflicts of near or stop-line crossings (TP) and far or free crossings (TA) were the only result. This lack of resolution can be partially attributed to the intrinsic variance of accident and conflict data. As shown, despite displaying relevant differences on the risk level of conflicts, the results were buried by their high variance and just suggested the potential of more detailed segmentations based on alternative criteria (saturation level on TP crossings and, less clearly, of pedestrian flows on TA crossings, as the sample was restricted to crossings with large pedestrian traffic).

The main result obtained, however, is the demonstration that the decision based criterion was very useful for selecting the segmentation of crossings and/or classification of conflict types and for justifying the best confidence level to be used in professional studies. Further research can extend the method to the development of parameters for other techniques and can also improve the method by including other effects in addition to accident reduction. 
Acknowledgements: especially to FAPESP-Foundation for Research Aid of the State of São Paulo and to CET/SP-Traffic Engineering Company of the City of São Paulo, for their collaboration in the original 1998 study, that gathered the data use in the research.

\section{REFERENCES}

[1]. ICTCT - INTERNATIONAL COMMITTEE ON TRAFFIC CONFLICT TECHNIQUE (1984) - The Malmö Study. Netherlands: Institute for Road Safety Research SWOV.

[2]. HYDÉN, C. (1987) - The Development of a Method for Traffic safety Evolution: The Swedish Traffic Conflicts Technique. Bulletin 70, Sweden.

[3]. MUhlard, N. (1988) Technique des Conflicts de Trafic - Manuel des L'Utilisateur, Synthese INRETS - Institute National de Recherche sur les Transports et leur Securite n.11, France.

[4]. BAGULEY, C. (1988) - Guidelines for the Traffic Conflict Technique. Hertford: IHT- Institute of Highway Transportation. (University College London Transport Studies Group)

[5]. PARKER Jr., M.R.; ZEGEER C.V. (1989) - Traffic Conflict Techniques for Safety and Operations Engineering Guide. Publication FHWA-IP88-026, FHWA - Federal Highway Administration, Department of Transportation, U.S.A.

[6]. PARKER Jr., M.R.; ZEGEER C.V. (1989) - Traffic Conflict Techniques for Safety and Operations Observer Manual. Publication FHWA-IP88-027, FHWA - Federal Highway Administration, Department of Transportation, U.S.A. 
[7]. GLAUZ, W.D.; BAUER, K.M.; \& MIGLETZ, D.J. (1985) - Expected Traffic Conflict Rates and Their Use in Predicting Accidents. TRB, Transportation Research Record, USA, n.1026.

[8]. GLAUZ, W.D. \& MIGLETZ, D.J. (1980) - Application of Traffic Conflict Analysis at Intersections. TRB, NCHRP Report, USA, n.219.

[9]. PIETRANTONiO, H. (1991) Manual of Procedures for Analysis of Traffic Conflicts at Intersections. IPT - Institute of Technological Research of the State of São Paulo. Transportation Engineering Group of the Division of Transportation Technology, São Paulo, Brazil (in portuguese).

[10]. GUEDES, E.; BRAGA, M.; PIETRANTONIO, H. (1997) - Initial Experiences with Traffic Conflict Technique in Brazil. Proceedings of the ICTCT97 Conference, Lund, Sweden.

[11]. ALMQVIST, S. (1998) - Introduction of the Swedish Traffic Conflict Technique - a Method for Assessing Traffic Safety. FAPESP-Fundação de Amparo à Pesquisa do Estado de São Paulo. (Relatório e Anexos Ia IV, Processo 97/12.154-0), São Paulo, Brazil.

[12]. PIETRAntoniO H. (1999) - Evaluation of the Swedish Traffic Conflict Analysis Technique Application to the Study of Pedestrian Safety Problems at Signalized Intersections of the City of São Paulo. EPUSP - Polytechnic School of the University of São Paulo. Working Report LEMT No.2/98, São Paulo, Brazil (in portuguese).

[13]. PIETRAntoniO, H.; TOURINHO, L.F.B. (2004) - Parameters for Evaluating Pedestrian Safety Problem in Signalized Intersections with the Traffic Conflict Analysis Technique -a Study in São Paulo, Brazil. Accepted for Presentation, $84^{\text {th }}$ Annual Meeting, Transportation Research Board, Washington, USA.

[14]. HAUER, E. (1975) - The Traffic Conflict Technique - Fundamental Issues. Publication 75-01. Department of Civil Engineering, University of Toronto, Ontario, Canada. 
[15]. COCHRAN, W. G. (1977) - Sampling Techniques. New York: John Wiley \& Sons. Third Edition.

[16]. HAUER; E. (1997) -Observational Before/After Studies in Road Safety: Estimating the Effect of Highway and Traffic Engineering Measures on Road Safety, Pergamon Press.

[17]. CAMERON, A. C.; TRIVEDI, P.K. (1998) -Regression Analysis of Count Data, Cambridge University Press. 


\section{LIST OF FIGURES}

Figure 1 - Gamma Distribution and the Limit Counts for $90^{\circ}$ and $95^{\circ}$ quantiles

Figure 2 - Pedestrian-Vehicle Conflict Types in Intersection Crossings

\section{LIST OF TABLES}

Table 1 - Approach for the Validation with Economic-based Weights and Representative Values

Table 2 - Basic Data on the Intersections and Crossings of the 1998 Study

Table 3 - Summary of Pedestrian Accidents and Recovered Police Reports in the Intersections

Table 4 - Summary of average daily conflicts (for the standard 11 hours period), São Paulo Study

a. General Data on the Intersections of the Studied Pedestrian Crossings

b. Data on the Studied Pedestrian Crossings of Each Intersection

Table 5 - Results on Normal Count Limits for Pedestrian-Vehicle Conflicts, São Paulo Study

a. Basic Results on Normal Conflict Count Limits based on the Sample

b. Results for Further Segmentation/Disaggregation of Normal Conflict Count Limits

Table 6 - Comparative Performance of Alternative Criteria for Abnormal Conflict Level Detection

Table 7 - Representative Factors for Converting Standard Period Conflict Counts to Annual Counts

Table 8 - Results on the Ratio of Pedestrians Accidents to Million Conflicts, São Paulo Study

a. Basic Results on the Ratio of Accidents to Million Conflicts based on the Sample

b. Further Results on the Ratio of Accidents to Million Conflicts based on the Sample 


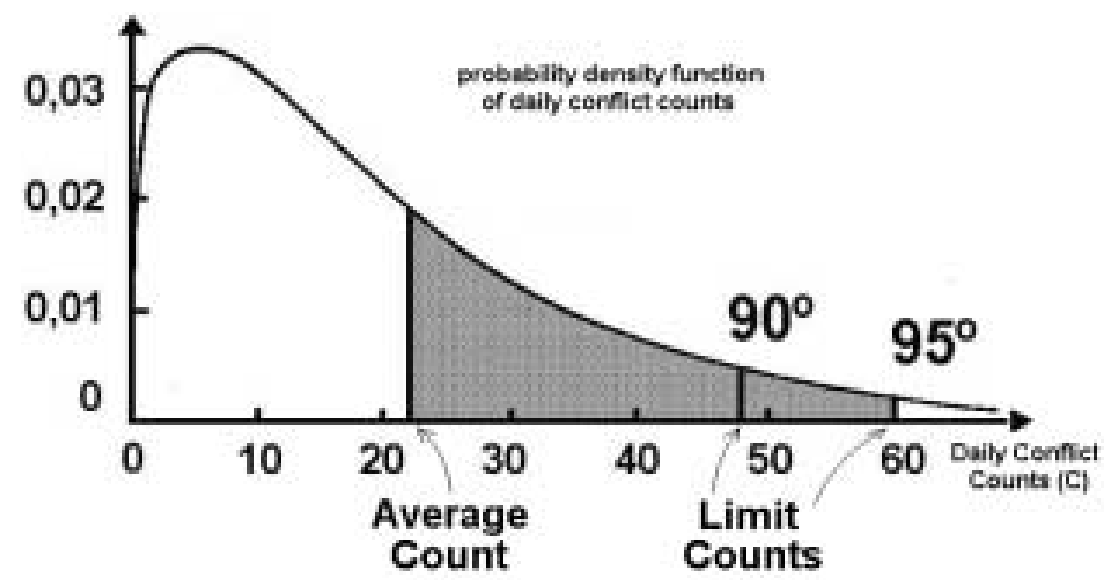

Figure 1 - Gamma Distribution and the Limit Counts for $90^{\circ}$ and $95^{\circ}$ quantiles. (based on [5]) 


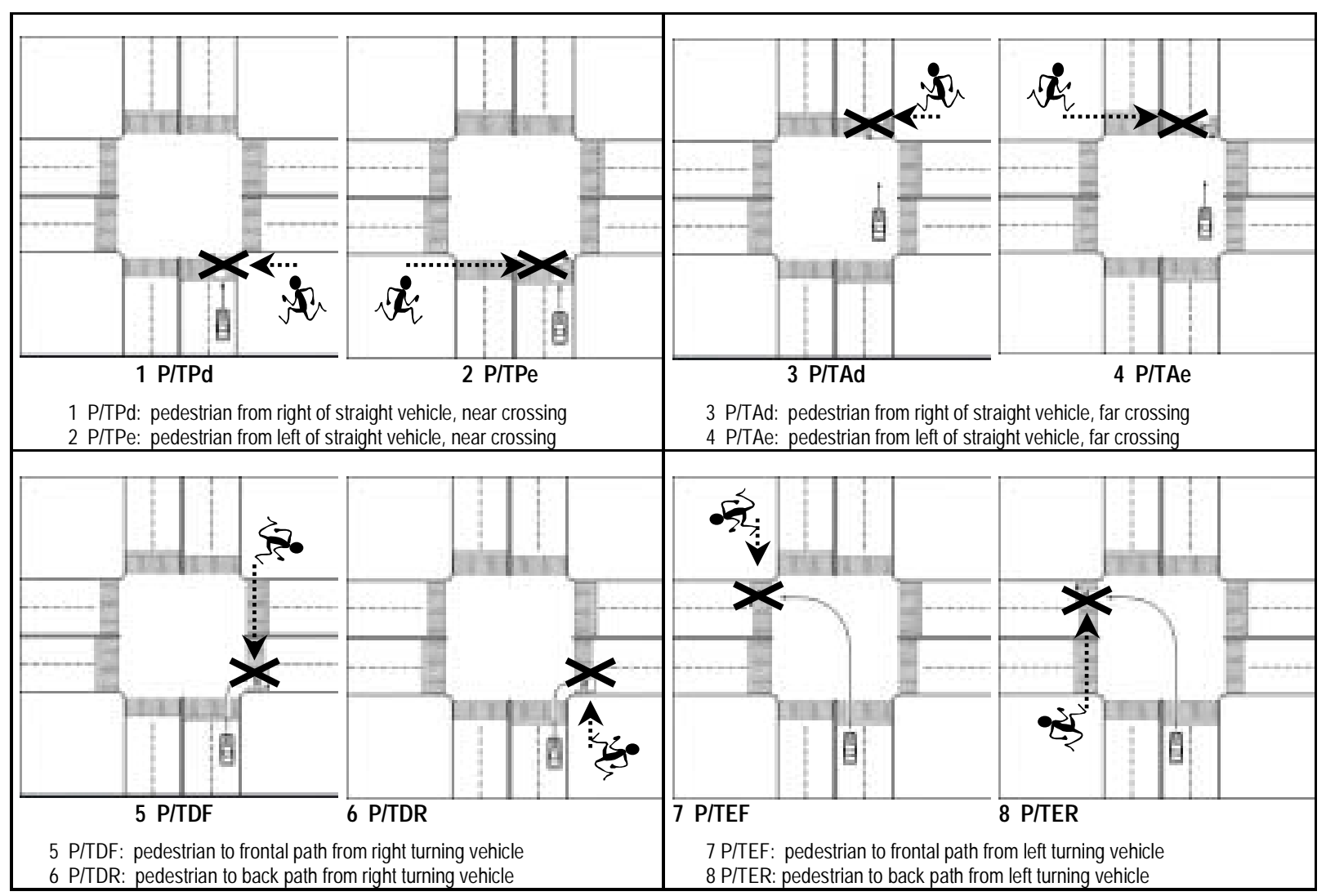

Figure 2 - Pedestrian-Vehicle Conflict Types in Intersection Crossings. 
Table 1 - Approach for the Validation with Economic-based Weights and Representative Values.

\begin{tabular}{|c|c|c|}
\hline $\begin{array}{l}\text { Size of the Validation } \\
\text { Sample }(\mathrm{N})\end{array}$ & $\begin{array}{l}\text { Sites with record of } \\
\text { Accidents (NB) }\end{array}$ & $\begin{array}{c}\text { Sites without record of } \\
\text { Accidents (NN) }\end{array}$ \\
\hline $\begin{array}{l}\text { Normal sites (NS) } \\
\text { with conflict counts } \\
\text { under Count Limits. }\end{array}$ & $\begin{array}{c}\text { Error Type I } \\
\text { NEI } \\
\text { (Neglected Risk) } \\
\text { Costs of Avoidable Accidents } \\
\end{array}$ & $\begin{array}{c}\text { HIT Type II } \\
\text { NEll } \\
\text { (Saving of Resources) } \\
\text { None (or benefit of alternative use) }\end{array}$ \\
\hline $\begin{array}{l}\text { Abnormal sites (NA) } \\
\text { with conflict counts } \\
\text { over Count Limits }\end{array}$ & $\begin{array}{c}\text { HIT Type I } \\
\text { NHI } \\
\text { (Detected Risk) } \\
\text { Benefit of Safety Improvement }\end{array}$ & $\begin{array}{c}\text { Error Type II } \\
\text { NHII } \\
\text { (Wasting of Resources) } \\
\text { Cost of Intervention } \\
\end{array}$ \\
\hline
\end{tabular}

- Type I Error

- Type II Error

- Type I Hit

- Type II Hit
Neglected risk: not selecting a site that has accident records in the sample information. The economic weight is related to the costs of avoidable accidents (e.g. $50 \%$ US $\$ 20.000 / a c c$ for direct costs of accidents).

Wasting of resources: spending money for treating a site without accident record in the sample information. The economic weight is related to the cost of usual treatments (e.g. US\$ $5.000 /$ crossing for small intervention).

Detected risk: selecting a site that has accident records in the sample information. The economic weight is related to the reduction of accidents less treatment cost (e.g. 50\% of US\$20.000/acc less US\$ $5.000 /$ crossing)

Saving of Resources: avoid spending money for sites without accident records in the sample information. The economic weight is null (or the average benefit of alternative uses of resources in other areas can be used) 
Table 2 - Basic Data on the Intersections and Crossings of the 1998 Study.

a. General Data on the Intersections of the Studied Pedestrian Crossings.

\begin{tabular}{|c|c|c|c|c|}
\hline Intersection & Identification of Roads & No.Approaches & No.Crossings & Obs.: \\
\hline Ip-SJ & $\begin{array}{c}\text { Ipiranga Av. } \\
\text { X São João Av. }\end{array}$ & 6 & $\begin{array}{c}\text { Both approaches of Ipiranga Av. have } \\
\text { the same direction of flow. }\end{array}$ \\
\hline Co-CP & $\begin{array}{l}\text { Consolação Av. } \\
\text { X Caio Prado St. }\end{array}$ & $2 \times 1$ & 7 & $\begin{array}{c}\text { Caio Prado St. split into two exiting } \\
\text { roads after the intersection }\end{array}$ \\
\hline FL-TS & $\begin{array}{c}\text { Faria Lima Av. } \\
\text { X Teodoro Sampaio St. }\end{array}$ & $2 \times 1$ & 7 & $\begin{array}{c}\text { Major right turn from the Teodoro } \\
\text { Sampaio St. before the intersection }\end{array}$ \\
\hline FM-VB & $\begin{array}{l}\text { Francisco Morato Av. } \\
\text { X Vital Brasil Av. }\end{array}$ & $3 \times 1$ & $\begin{array}{c}\text { One-way busway to the CBD at the } \\
\text { center of the Francisco Morato Av. }\end{array}$ \\
\hline
\end{tabular}

b. Data on the Studied Pedestrian Crossings of Each Intersection.

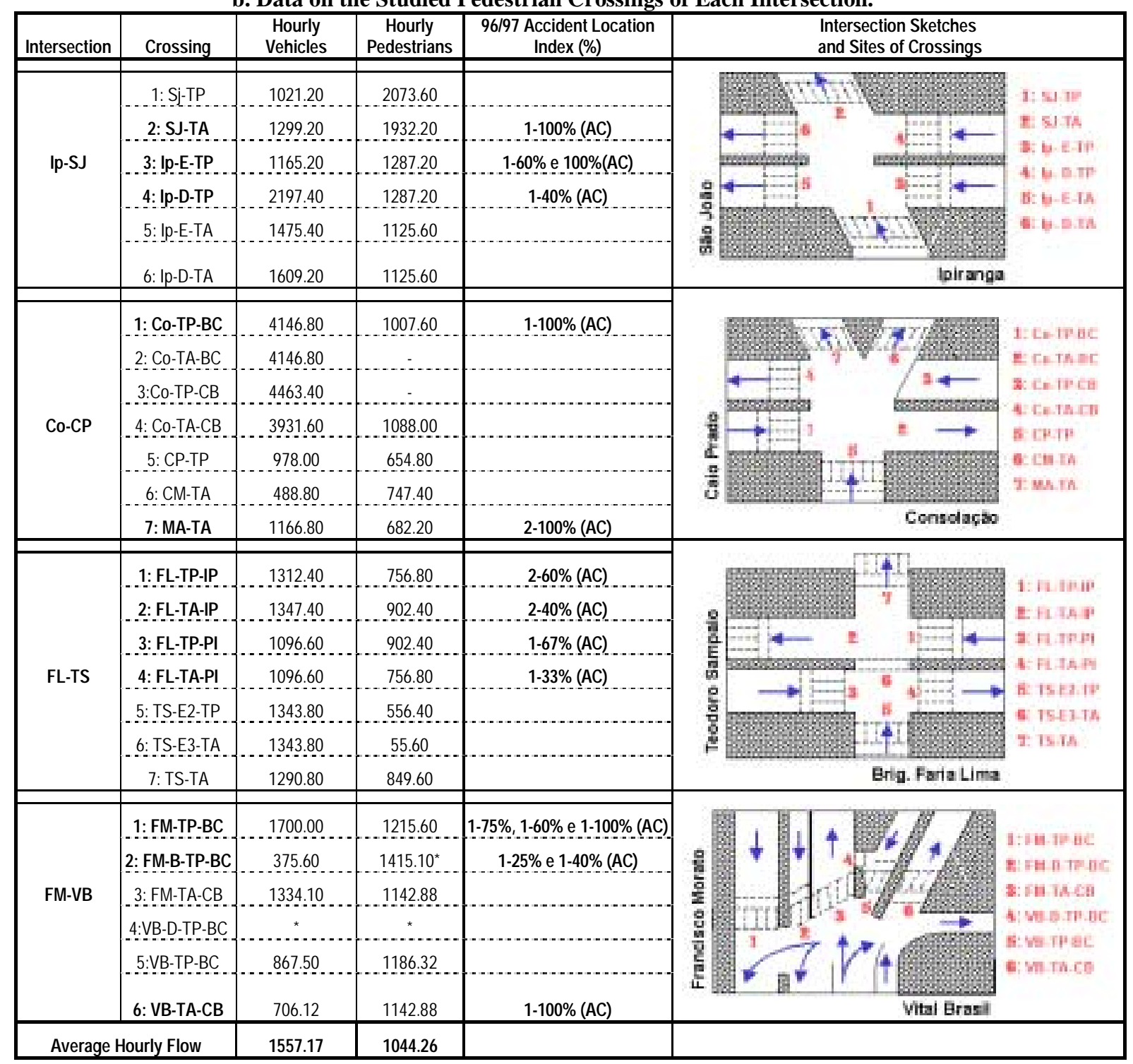


Table 3 - Summary of Pedestrian Accidents and Recovered Police Reports in the Intersections.

\begin{tabular}{|c|c|c|c|c|c|c|c|c|}
\hline \multicolumn{9}{|c|}{ Pedestrian Accidents - 1996/1997 } \\
\hline \multicolumn{2}{|c|}{ Intersection } & Year & $\begin{array}{c}\text { Total } \\
\text { Ped.Acc. }\end{array}$ & $\begin{array}{l}\text { Work } \\
\text { Days }\end{array}$ & $\begin{array}{l}\text { Week } \\
\text { Ends }\end{array}$ & $\begin{array}{l}\text { Recovered in } \\
\text { each year }\end{array}$ & $\begin{array}{l}\text { Total and \% } \\
\text { Recovered }\end{array}$ & $\begin{array}{c}\text { in workday } \\
\text { standard period }\end{array}$ \\
\hline \multirow{4}{*}{ 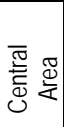 } & \multirow[b]{2}{*}{ Ip-SJ } & 96 & 8 & 7 & 1 & 3 & \multirow{2}{*}{$\begin{array}{c}8 \\
(53 \%)\end{array}$} & 1 \\
\hline & & 97 & 7 & 4 & 3 & 5 & & 2 \\
\hline & \multirow[b]{2}{*}{ Co-CP } & 96 & 5 & 2 & 3 & 3 & \multirow{2}{*}{$\begin{array}{c}7 \\
(58 \%)\end{array}$} & 1 \\
\hline & & 97 & 7 & 7 & 0 & 4 & & 2 \\
\hline \multirow{4}{*}{ 善岕 } & \multirow[b]{2}{*}{ FM-VB } & 96 & 8 & 7 & 1 & 3 & \multirow{2}{*}{$\begin{array}{c}6 \\
(35 \%)\end{array}$} & 1 \\
\hline & & 97 & 9 & 7 & 2 & 3 & & 3 \\
\hline & \multirow[b]{2}{*}{ FL-TS } & 96 & 7 & 4 & 3 & 4 & \multirow{2}{*}{$\begin{array}{c}5 \\
(50 \%)\end{array}$} & 2 \\
\hline & & 97 & 3 & 3 & 0 & 1 & & 1 \\
\hline & & Total & 54 & 41 & 13 & 26 & & 13 \\
\hline
\end{tabular}

Source:Accident Records- Police of the State of São Paulo 
Table 4 - Summary of average daily conflicts (for the standard 11 hours period), São Paulo Study.

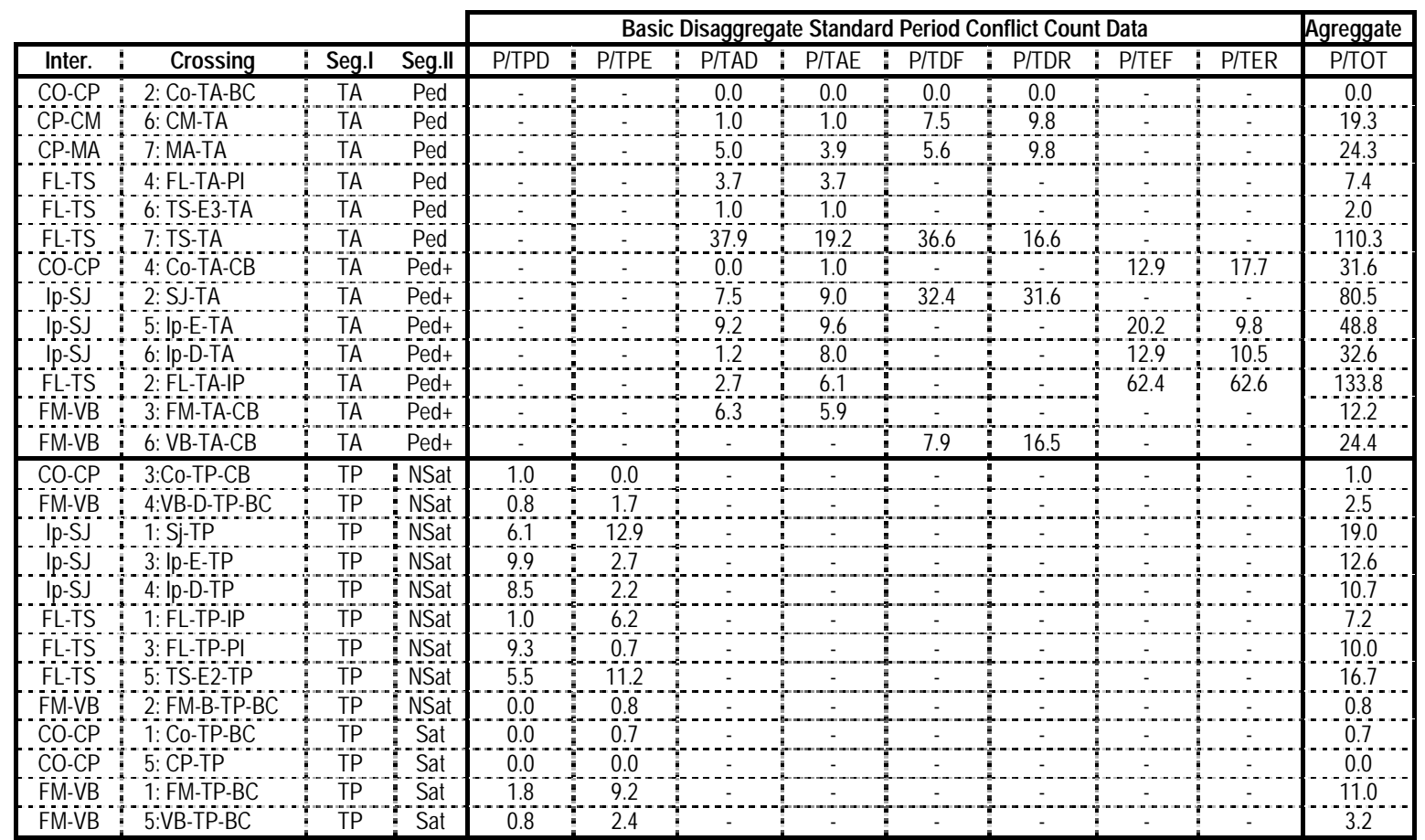

Note: Cells with - can not have conflicts of the related type. Zero counts are explicitly indicates as 0,0 (no count during the surveyed and standard periods). 
Table 5 - Results on Normal Count Limits for Pedestrian-Vehicle Conflicts, São Paulo Study.

a. Basic Results for Count Limits based on the Sample (Confidence Levels CL=75\%, 90\%, $95 \%$ ).

\begin{tabular}{|c|c|c|c|c|c|c|c|c|c|c|}
\hline Segmentation & $\begin{array}{l}\text { All- } \\
\text { Aggregate }\end{array}$ & \begin{tabular}{|l} 
All- \\
2Types
\end{tabular} & & \begin{tabular}{|l}
$\mathrm{TA} / \mathrm{TP}-$ \\
Aggregate
\end{tabular} & $\begin{array}{l}\text { TA/TP- } \\
2 \text { Types }\end{array}$ & & $\begin{array}{l}\text { TA/TP- } \\
\text { 4Types }\end{array}$ & & & \\
\hline Conflict Type & Aggregate & $\mathrm{P} / \mathrm{VA}$ & $\overline{\mathrm{P} / \mathrm{VT}}$ & Aggregate & $\mathrm{P} / \mathrm{VA}$ & $\bar{P} / \mathrm{VT}$ & $\mathrm{P} / \mathrm{TP}$ & $\mathrm{P} / \mathrm{TA}$ & $\mathrm{P} / \mathrm{TD}$ & $\bar{P} / \mathrm{TE}$ \\
\hline CrossingType & All & \multicolumn{2}{|l|}{ All } & $\mathrm{TA}$ & \multicolumn{2}{|l|}{ TA } & \multicolumn{4}{|l|}{ All-TA } \\
\hline average & \multirow{11}{*}{$\begin{array}{c}23.9 \\
1162.1 \\
31.6 \\
65.0 \\
92.4\end{array}$} & \multirow{11}{*}{$\begin{array}{c}9.6 \\
135.6 \\
13.1 \\
24.2 \\
33.0\end{array}$} & \multirow{11}{*}{$\begin{array}{c}38.3 \\
1263.9 \\
53.0 \\
85.0 \\
108.9\end{array}$} & 40.6 & 12.0 & 383 & & 12.0 & 29.1 & 52.3 \\
\hline variance & & & & 1781.5 & 237.7 & 1263.9 & & 237.7 & 598.9 & 2362.9 \\
\hline$C L=75 \%$ & & & & 56.2 & 16.3 & 53.0 & & 16.3 & 39.9 & 72.3 \\
\hline $90 \%$ & & & & 95.2 & 31.1 & 85.0 & & 31.1 & 61.5 & 116.1 \\
\hline $95 \%$ & & & & 125.0 & 43.0 & 108.9 & & 43.0 & 77.3 & 148.8 \\
\hline CrossingType & & & & TP & TP & & All-TP & & & \\
\hline average & & & & 7.3 & 7.3 & & 7.3 & & & \\
\hline variance & & & & 42.0 & 42.0 & & 42.0 & & & \\
\hline$C L=75 \%$ & & & & 10.1 & 10.1 & & 10.1 & & & \\
\hline $90 \%$ & & & & 15.9 & 15.9 & & 15.9 & & & \\
\hline $95 \%$ & & & & 20.2 & 20.2 & & 20.2 & & & \\
\hline
\end{tabular}

b. Further Results for Count Limits based on the Sample (Confidence Levels CL=75\%, 90\%, 95\%).

\begin{tabular}{|c|c|c|c|c|c|c|c|c|c|c|c|}
\hline \multirow{2}{*}{\begin{tabular}{|l} 
Segmentation \\
Conflict Type
\end{tabular}} & \multirow{2}{*}{\begin{tabular}{|l|} 
Ped/+/N/S \\
Aggregate \\
Aggregate
\end{tabular}} & \multicolumn{2}{|c|}{$\begin{array}{l}\text { Ped///N/Sat- } \\
2 \text { Types }\end{array}$} & \multicolumn{8}{|l|}{$\begin{array}{l}\text { TA/TP- } \\
\text { 8Types }\end{array}$} \\
\hline & & $\mathrm{P} / \mathrm{VA}$ & $\mathrm{P} / \mathrm{VT}$ & P/TPD & P/TPE & $\mathrm{P} / \mathrm{TAD}$ & $\mathrm{P} / \mathrm{TAE}$ & $\mathrm{P} / \mathrm{TDF}$ & P/TDR & $\mathrm{P} / \mathrm{TEF}$ & P/TER \\
\hline CrossingType & TA-Ped & \multicolumn{2}{|l|}{ TA-Ped } & \multicolumn{8}{|l|}{$\overline{A l l-T A}$} \\
\hline average & 27.2 & 12.9 & 21.5 & & & 6.3 & 5.7 & 15.0 & 14.1 & 27.1 & 25.2 \\
\hline variance & 1748.7 & 480.8 & 507.3 & & & 108.3 & 29.1 & 237.9 & 110.8 & 565.7 & 636.1 \\
\hline$C L=75 \%$ & 34.8 & 15.6 & 29.8 & & & 7.7 & 7.9 & 20.8 & 19.1 & 37.3 & 34.9 \\
\hline $90 \%$ & 76.1 & 37.3 & 50.6 & & & 18.0 & 12.8 & 35.0 & 28.1 & 58.5 & 58.0 \\
\hline $95 \%$ & 110.9 & 56.3 & 66.5 & & & 27.0 & 16.4 & 45.8 & 34.6 & 74.1 & 75.5 \\
\hline CrossingType & TA-Ped+ & \multicolumn{2}{|l|}{ TA-Ped+ } & & & & & & & & \\
\hline average & 52.0 & 11.1 & 49.6 & & & & & & & & \\
\hline variance & 1775.3 & 40.1 & 1591.8 & & & & & & & & \\
\hline$C L=75 \%$ & 71.1 & 14.5 & 67.8 & & & & & & & & \\
\hline $90 \%$ & 107.9 & 19.6 & 102.6 & & & & & & & & \\
\hline $95 \%$ & 134.8 & 23.1 & 127.9 & & & & & & & & \\
\hline CrossingType & TP-NSat & \multicolumn{2}{|l|}{ TP-NSat } & \multicolumn{8}{|l|}{ All-TP } \\
\hline average & 8.9 & 9.9 & & \multirow{11}{*}{$\begin{array}{c}3.4 \\
14.8 \\
4.8 \\
8.4 \\
11.1\end{array}$} & \multirow{11}{*}{$\begin{array}{c}3.9 \\
20.0 \\
5.4 \\
9.6 \\
12.9\end{array}$} & & & & & & \\
\hline variance & 44.1 & 40.3 & & & & & & & & & \\
\hline $\mathrm{CL}=75 \%$ & 12.1 & 13.2 & & & & & & & & & \\
\hline $90 \%$ & 17.8 & 18.4 & & & & & & & & & \\
\hline $95 \%$ & 21.9 & 22.1 & & & & & & & & & \\
\hline CrossingType & TP-Sat & \multicolumn{2}{|l|}{ TP-Sat } & & & & & & & & \\
\hline average & 3.7 & 3.7 & & & & & & & & & \\
\hline variance & 25.4 & 25.4 & & & & & & & & & \\
\hline$C L=75 \%$ & 5.0 & 5.0 & & & & & & & & & \\
\hline $90 \%$ & 9.9 & 9.9 & & & & & & & & & \\
\hline $95 \%$ & 13.9 & 13.9 & & & & & & & & & \\
\hline
\end{tabular}


Table 6 - Comparative Performance of Alternative Criteria for Detection of Abnormal Conflict Level.

\begin{tabular}{|c|c|c|c|c|c|c|c|c|c|c|c|}
\hline $\begin{array}{l}\text { Confidence } \\
\text { Level }\end{array}$ & & $\begin{array}{l}\text { All- } \\
\text { Aggregate }\end{array}$ & $\begin{array}{l}\text { TA/TP- } \\
\text { Aggregate }\end{array}$ & \multicolumn{2}{|c|}{$\begin{array}{l}\text { Ped/+/N/Sat- All- } \\
\text { Aggregate } \quad 2 \text { Types }\end{array}$} & $\begin{array}{l}\text { TA/TP- } \\
2 \text { Types } \\
\end{array}$ & \multicolumn{2}{|c|}{$\mathrm{Ped} /+/ \mathrm{N} / \mathrm{Sat}-\mathrm{TA} / \mathrm{TP}-$} & \multicolumn{2}{|c|}{$\mathrm{Ped} /+/ \mathrm{N} /$ Sat- TA/TP- } & $\begin{array}{l}\mathrm{Ped} /+/ \mathrm{N} / \mathrm{Sat}- \\
8 \text { Types }\end{array}$ \\
\hline \multirow[t]{14}{*}{$75 \%$} & NoErr1 & 10 & 7 & 8 & 10 & 7 & 8 & 7 & 9 & 5 & 6 \\
\hline & NoHit1 & 2 & 5 & 4 & 2 & 5 & 4 & 5 & 3 & 7 & 6 \\
\hline & $\% H i t 1$ & $16.67 \%$ & $41.67 \%$ & $33.33 \%$ & $16.67 \%$ & $41.67 \%$ & $33.33 \%$ & $41.67 \%$ & $25.00 \%$ & $58.33 \%$ & $50.00 \%$ \\
\hline & NoErr2 & 3 & 3 & 3 & 4 & 4 & 4 & 4 & 4 & 5 & 5 \\
\hline & NoHit2 & 11 & 11 & 11 & 10 & 10 & 10 & 10 & 10 & 9 & 9 \\
\hline & $\%$ Hit2 & $78.57 \%$ & $78.57 \%$ & $78.57 \%$ & $71.43 \%$ & $71.43 \%$ & $71.43 \%$ & $71.43 \%$ & $71.43 \%$ & $64.29 \%$ & $64.29 \%$ \\
\hline & AccE1 & 11 & 7 & 7,5 & 11 & 7 & 7,5 & 7 & 9 & 5,5 & 6,5 \\
\hline & $\mathrm{AccH} 1$ & 2 & 6 & 5,5 & 2 & 6 & 5,5 & 6 & 4 & 7,5 & 6,5 \\
\hline & DE & -5 & 20 & 20 & -10 & 15 & 15 & 15 & 5 & 15 & 10 \\
\hline & TA-Ped & -5 & -5 & -5 & -5 & -5 & -5 & -5 & -5 & -5 & -5 \\
\hline & TA-Ped+ & 0 & 10 & 10 & 5 & 5 & 5 & 5 & 5 & 0 & 0 \\
\hline & TP-NSat & 0 & 0 & 0 & -10 & 0 & 0 & 0 & -10 & 5 & 0 \\
\hline & TP-Sat & 0 & 15 & 15 & 0 & 15 & 15 & 15 & 15 & 15 & 15 \\
\hline & $\mathrm{RE}$ & $-7.14 \%$ & $28.57 \%$ & $28.57 \%$ & $-14.29 \%$ & $21.43 \%$ & $21.43 \%$ & $21.43 \%$ & $7.14 \%$ & $21.43 \%$ & $14.29 \%$ \\
\hline \multirow[t]{14}{*}{$90 \%$} & NoErr1 & 10 & 11 & 10 & 11 & 11 & 10 & 10 & 10 & 7 & 10 \\
\hline & NoHit1 & 2 & 1 & 2 & 1 & 1 & 2 & 2 & 2 & 5 & 2 \\
\hline & $\%$ Hit1 & $16.67 \%$ & $8.33 \%$ & $16.67 \%$ & $8.33 \%$ & $8.33 \%$ & $16.67 \%$ & $16.67 \%$ & $16.67 \%$ & $41.67 \%$ & $16.67 \%$ \\
\hline & NoErr2 & 1 & 3 & 2 & 1 & 3 & 2 & 3 & 2 & 3 & 3 \\
\hline & NoHit2 & 13 & 11 & 12 & 13 & 11 & 12 & 11 & 12 & 11 & 11 \\
\hline & $\%$ Hit2 & $92.86 \%$ & $78.57 \%$ & $85.71 \%$ & $92.86 \%$ & $78.57 \%$ & $85.71 \%$ & $78.57 \%$ & $85.71 \%$ & $78.57 \%$ & $78.57 \%$ \\
\hline & AccE1 & 11 & 12 & 10 & 12 & 12 & 10 & 11 & 10 & 8,5 & 10 \\
\hline & $\mathrm{AccH} 1$ & 2 & 1 & 3 & 1 & 1 & 3 & 2 & 3 & 4,5 & 3 \\
\hline & DE & 5 & -10 & 10 & 0 & -10 & 10 & -5 & 10 & 5 & 5 \\
\hline & TA-Ped & -5 & -5 & -5 & -5 & -5 & -5 & -5 & -5 & -5 & -5 \\
\hline & TA-Ped+ & 10 & 5 & 5 & 5 & 5 & 5 & 10 & 5 & 10 & 5 \\
\hline & TP-NSat & 0 & -10 & -5 & 0 & -10 & -5 & -10 & -5 & 0 & -10 \\
\hline & TP-Sat & 0 & 0 & 15 & 0 & 0 & 15 & 0 & 15 & 0 & 15 \\
\hline & RE & $7.14 \%$ & $-14.29 \%$ & $14.29 \%$ & $0.00 \%$ & $-14.29 \%$ & $14.29 \%$ & $-7.14 \%$ & $14.29 \%$ & $7.14 \%$ & $7.14 \%$ \\
\hline \multirow[t]{14}{*}{$95 \%$} & NoErr1 & 11 & 11 & 12 & 11 & 11 & 12 & 12 & 12 & 12 & 12 \\
\hline & NoHit1 & 1 & 1 & 0 & 1 & 1 & 0 & 0 & 0 & 0 & 0 \\
\hline & $\%$ Hit1 & $8.33 \%$ & $8.33 \%$ & $0.00 \%$ & $8.33 \%$ & $8.33 \%$ & $0.00 \%$ & $0.00 \%$ & $0.00 \%$ & $0.00 \%$ & $0.00 \%$ \\
\hline & NoErr2 & 1 & 0 & 0 & 1 & 1 & 1 & 1 & 1 & 2 & 1 \\
\hline & NoHit2 & 13 & 14 & 14 & 13 & 13 & 13 & 13 & 13 & 12 & 13 \\
\hline & $\%$ Hit2 & $92.86 \%$ & $100.00 \%$ & $100.00 \%$ & $92.86 \%$ & $92.86 \%$ & $92.86 \%$ & $92.86 \%$ & $92.86 \%$ & $85.71 \%$ & $92.86 \%$ \\
\hline & AccE1 & 12 & 12 & 13 & 12 & 12 & 13 & 13 & 13 & 13 & 13 \\
\hline & $\mathrm{AccH} 1$ & 1 & 1 & 0 & 1 & 1 & 0 & 0 & 0 & 0 & 0 \\
\hline & $\mathrm{DE}$ & 0 & 5 & 0 & 0 & 0 & -5 & -5 & -5 & -10 & -5 \\
\hline & TA-Ped & -5 & 0 & 0 & -5 & -5 & -5 & -5 & -5 & -5 & -5 \\
\hline & TA-Ped+ & 5 & 5 & 0 & 5 & 5 & 0 & 0 & 0 & 0 & 0 \\
\hline & TP-NSat & 0 & 0 & 0 & 0 & 0 & 0 & 0 & 0 & -5 & 0 \\
\hline & TP-Sat & 0 & 0 & 0 & 0 & 0 & 0 & 0 & 0 & 0 & 0 \\
\hline & $\mathrm{RE}$ & $0.00 \%$ & $7.14 \%$ & $0.00 \%$ & $0.00 \%$ & $0.00 \%$ & $-7.14 \%$ & $-7.14 \%$ & $-7.14 \%$ & $-14.29 \%$ & $-7.14 \%$ \\
\hline
\end{tabular}


Table 7 - Representative Factors for Converting Standard Period Conflict Counts to Annual Counts.

\begin{tabular}{|c|c|c|c|c|}
\cline { 2 - 5 } \multicolumn{1}{c|}{} & \multicolumn{2}{c|}{ dry } & pavement & \multicolumn{2}{c|}{ wet } & pavement \\
\cline { 2 - 5 } \multicolumn{1}{c|}{} & Std/Day & Days/Year & Std/Day & Days/Year \\
\hline Work Days & $70 \%$ & $4 / 7$ of 365 & $70 \%$ & $1 / 7$ of 365 \\
Week Ends & $70 \%$ & $3 / 14$ of 365 & $70 \%$ & $1 / 14$ of 365 \\
\hline
\end{tabular}


Table 8 - Results on the Ratio of Pedestrians Accidents to Million Conflicts, São Paulo Study.

a. Basic Results for the Ratio of Accidents to Million Conflicts based on the Sample.

\begin{tabular}{|c|c|c|c|c|}
\hline & $\begin{array}{l}\text { Aggregate- } \\
\text { All }\end{array}$ & $\begin{array}{l}\text { Aggregate- } \\
\text { TA }\end{array}$ & $\begin{array}{l}\text { Aggregate- } \\
\text { TP }\end{array}$ & $\begin{array}{r}\text { diff: } \\
\text { TA/TP }\end{array}$ \\
\hline No.Sites & 26 & 13 & 13 & \\
\hline Total Accidents & 13.0 & 5.5 & 7.5 & \\
\hline $\mathrm{R}(\mathrm{Ac} / \mathrm{MC})$ & 35.0387 & 17.5065 & 131.9247 & -114.4182 \\
\hline Standard Dev. & 12.7482 & 8.0006 & 51.0302 & 51.6536 \\
\hline Quasi-t & 2.7485 & 2.1882 & 2.5852 & -2.2151 \\
\hline Coef.Variation & $36.38 \%$ & $45.70 \%$ & $38.68 \%$ & \\
\hline Adjusted Ratio * & 78.1632 & 39.0531 & 294.2936 & \\
\hline $\mathrm{RR}(\mathrm{Ay} / \mathrm{Ch})$ & 0.2562 & 0.1280 & 0.9646 & \\
\hline MeanAccidents & 0.50 & 0.42 & 0.58 & \\
\hline MeanAbsErrorR ** & 0.67 & 0.47 & 0.61 & \\
\hline MaxAbsErrorR ** & 2.30 & 1.75 & 1.49 & \\
\hline MeanAbsErrorS ** & 0.54 & 0.52 & 0.56 & \\
\hline MaxAbsErrorS ** & 1.50 & 1.58 & 1.42 & \\
\hline
\end{tabular}

* The adjusted ratio is the final parameter for practical purposes as it includes a general correction factor (actually 29/13=2.23), for the partial set of accident reports recovered and used in the analysis.

** ErrorR and ErrorS (Mean or Max) are errors of predicted against observed accident frequencies using the ratio of accidents to conflict counts and the sample average of accident counts, respectively.

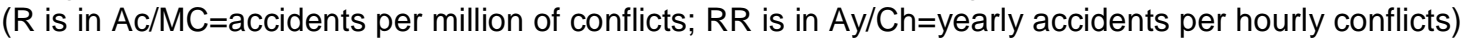

b. Further Results for the Ratio of Accidents to Million Conflicts based on the Sample.

\begin{tabular}{|c|c|c|c|c|c|c|}
\hline & $\begin{array}{l}\text { Aggregate- } \\
\text { TA-Ped }\end{array}$ & $\begin{array}{l}\text { Aggregate- } \\
\text { TA-Ped+ }\end{array}$ & $\begin{array}{r}\text { diff:TA- } \\
\text { Ped/Ped+ }\end{array}$ & $\begin{array}{l}\text { Aggregate- } \\
\text { TP-NSat }\end{array}$ & egate- & $\begin{array}{r}\text { diff:TP- } \\
\text { NSat/Sat }\end{array}$ \\
\hline No.Sites & 6 & 7 & & 9 & 4 & \\
\hline Total Accidents & 2.5 & 3.0 & & 4.5 & 3.0 & \\
\hline $\mathrm{R}(\mathrm{Ac} / \mathrm{MC})$ & 25.6902 & 13.8341 & 11.8560 & 93.8058 & 337.8689 & -244.0631 \\
\hline Standard Dev. & 26.9684 & 6.9915 & 27.8599 & 43.8764 & 142.4120 & 149.0179 \\
\hline Quasi-t & 0.9526 & 1.9787 & 0.4256 & 2.1380 & 2.3725 & -1.6378 \\
\hline Coef.Variation & $104.98 \%$ & $50.54 \%$ & & $46.77 \%$ & $42.15 \%$ & \\
\hline Adjusted Ratio * & 57.3089 & 30.8608 & & 209.2592 & 753.7075 & \\
\hline $\mathrm{RR}(\mathrm{Ay} / \mathrm{Ch})$ & 0.1878 & 0.1011 & & 0.6859 & 2.4703 & \\
\hline MeanAccidents & 0.42 & 0.43 & & 0.50 & 0.75 & \\
\hline MeanAbsErrorR ** & 0.67 & 0.32 & & 0.52 & 0.43 & \\
\hline MaxAbsErrorR ** & 1.69 & 0.80 & & 1.06 & 0.86 & \\
\hline MeanAbsErrorS ** & 0.56 & 0.49 & & 0.44 & 0.75 & \\
\hline MaxAbsErrorS ** & 1.58 & 0.57 & & 1.00 & 1.25 & \\
\hline
\end{tabular}

* The adjusted ratio is the final parameter for practical purposes as it includes a general correction factor (actually 29/13=2.23), for the partial set of accident reports recovered and used in the analysis.

** ErrorR and ErrorS (Mean or Max) are errors of predicted against observed accident frequencies using the ratio of accidents to conflict counts and the sample mean of accident counts, respectively.

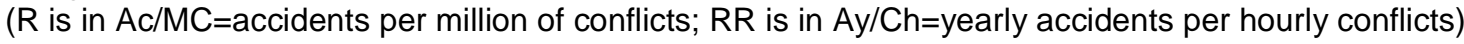

